\title{
ROSSO FIORENTINO, UN PINTOR MANIERISTA
}

\section{Por Teresa del Conde}

La reinterpretación y valoración del manierismo, considerado como un estilo artístico definido que se inicia en Italia a partir de la tercera década del siglo xvr, ha sido llevada a cabo paralelamente al desarrollo de los movimientos expresionistas y de las tendencias hacia la representación no figurativa que promovieron la revolución artística impllcita en las corrientes post-impresionistas.

El arte actual se caracteriza, fundamentalmente, por la ruptura con la representación naturalista de la realidad. Este proceso de oposición hacia el arte como mimesis, ha suscitado el interés por la revisión y el estudio de todos aquellos movimientos artísticos que en una forma u otra, han presentado elementos antagónicos a las normas que conforman el concepto de lo clásico, término que se entiende sólo en contraposición con su opuesto, que seria lo anti-clásico.

El manierismo tiene una fase anti-clásica y anti-naturalista que se relaciona históricamente con un periodo crítico, abundante en situaciones contradictorias, en el cual se produjo un derrumbamiento en la escala de los valores tradicionalmente aceptados. Esta fase conforma una etapa ilustrativa del "malestar en la cultura", denominación freudiana que Arnold Hauser estudia en "El arte en el mundo alienado", capítulo que incluye en su famoso tratado sobre el manierismo. ${ }^{1}$

El manierismo es un movimiento surgido en pleno Renacimiento, por lo tanto convive inicialmente con el espiritu clásico del estilo renacentista y posteriormente con tendencias barrocas, surgidas del movimiento de la Contrarreforma. "Maniera", según Giorgio Vasari, es una palabra que equivale a estilo, o sea a ejercicio del arte de acuerdo a determinados esquemas. Sin embargo, el manierismo es un movimiento sumamente complicado que no puede reducirse a un fenómeno de decadencia artística manifestado en ejercicio del arte como rutina. Para profundizarlo, hay que sondear en la actitud que los artistas activos en Italia durante la tercera década del siglo xvi asumfan frente al arte clásico del Renacimiento. La actitud de dichos artistas, que representan el manierismo en su fase más caracteristica, es siempre dialéctica: 1965.

1 Arnold Hauser. El Manierismo, crisis del Renacimiento. Guadarrama, Madrid, 
tanto en las letras cuanto en las artes plásticas, se basa en la imitación y en la simultánea distorsión de los modelos clásicos; este fenómeno no se da como una resultante de la falta de creatividad, sino en virtud de la presencia de un espíritu nuevo, a la vez derivado y antagónico al clasicismo renacentista. Así, la imitación de los modelos clásicos se presenta en el manierismo como una medida defensiva, necesaria para evitar el caos completo; en tanto que la agudización, las alteraciones y la estilización de los principios formales clásicos, expresan la vitalidad del impulso creativo y evitan la caída en un formalismo sin alma.

Generalizando, el Renacimiento clásico desarrolla la composición artística bajo las siguientes características:

a) Unidad coherente del espacio y de las proporciones.

b) Limitación de la representación a un motivo principal.

c) Ordenamiento totalitario de la composición.

d) Correspondencia de las partes con el todo y de las partes entre sí.

La misma aversión a todo lo que escapa al orden, impera en una serie de aspectos que caracterizan la vida en pleno Renacimiento; éstos se manifiestan en la organización del trabajo, en las técnicas comerciales y bancarias, en los métodos de gobierno y hasta en la diplomacia. Sin embargo, todo este periodo, a pesar de su aparente solidez, contiene los principios de su propia destrucción, es una "sutil cresta", que apenas alcanzada, ya presenta elementos de disolución y está en cierto modo superada, como afirma $\mathrm{H}$. Wolfflin. ${ }^{2}$

La brevedad del tiempo en que imperaron los principios clásicos y el rigor formal, puede explicarse porque el equilibrio que encontró su expresión artística en el clasicismo, fue desde sus inicios más bien un ideal perseguido que una realización sólida, y el Renacimiento por ello mismo siguió siendo hasta el final una época de grandes cambios, que no adoptó soluciones permanentes.

Entre los artistas que representan el movimiento manierista en su forma más auténtica, existieron personalidades que tanto por sus temperamentos, cuanto por el decurso de sus vidas, plasmaron formas de existencia muy singulares, que tienen algunos rasgos comunes: la actitud ambivalente ante el arte, la religión y la vida en general, la desmesurada forma de respuesta ante estimulos cuyas naturalezas no guar-

$2 \mathrm{H}$. Wolfflin. Conceptos fundamentales en la historia del arte. Espasa-Calpe, Madrid, 1952, p. 24 y ss. 
dan correlación con los efectos que produjeron y a la vez la presencia de un potencial creativo intenso que parece haberse alimentado a partir de la angustia. Miguel Angel, manierista de sí mismo, Pontormo, E1 Parmigianino y Rosso Fiorentino están entre ellos.

Miguel Angel, invertido, de carácter mezquino, con una creatividad raras veces igualada en la historia del arte, vivió siempre atormentado por escrúpulos y por motivos religiosos, además de que posiblemente sus ciclos hayan correspondido a los de una personalidad maníacodepresiva. Pontormo tenía un carácter extraño, hipersensitivo y altamente neurótico, la mayor parte de su vida fue un tremendo solitario y sin embargo dejó un conjunto de obras que se cuentan entre las más originales del periodo. Parmigianino fue uno de los artistas más finos de su tiempo, era un hombre elegante, de excelente posición, aceptado socialmente como persona y como artista, a pesar de lo cual a la edad de treinta años se tornó en un ser radicalmente opuesto a lo que era, casi en un salvaje, presentando un alto grado de descuido hacia su ser físico, además de que su interés obsesivo por ciertas disciplinas, como la alquimia, le restaron tiempo a su obra creativa. De Rosso Fiorentino habremos de ocuparnos en forma más amplia.

El verdadero nombre de Rosso era Gian Battista di Jacopo di Gasparre, se le conoce como Rosso de Florencia debido a la coloración rojiza de sus cabellos y al sitio de su nacimiento. Nació en 1495 y a los 22 años era ya pintor de cierta reputación. Cuando tenía esa edad se trasladó a Roma, donde estuvo en estrecho contacto con Miguel Ángel. Rosso no constituye una excepción en cuanto a receptividad del influjo miguelangelesco, antes al contrario, a partir de su conocimiento de la obra del poderoso florentino, el lenguaje formal de Rosso queda profundamente vinculado a la vertiente manierista de Miguel Ángel.

En 1527 tuvo lugar un hecho histórico importante: el saqueo de Roma, realizado por las tropas imperiales españolas, a consecuencia de lo cual la autoridad del Vaticano y la preponderancia artística de Roma se vieron en menoscabo. Muchos de los artistas que se encontraban en la ciudad cuando ocurrió el suceso, que duró una semana, salieron buscando refugio en otras regiones. Rosso inició en este tiempo un largo peregrinaje por varias ciudades italianas: Perugia, Borgo di San Sapolcro, Cittá di Castello, Arezzo, hasta que decidió salir de Italia estableciéndose definitivamente en Fontaineblau como pintor de Francisco I de Francia, el año de 1530. 
El arte de Rosso, como el de la mayoría de los manieristas, conjuga la influencia de varios artistas del Renacimiento, todos ellos discímiles entre si, como son Leonardo da Vinci, Fra Bartolomeo, Andrea del Sarto, los pintores y grabadores alemanes, y sobre todos ellos Miguel Angel. Puede parecer paradójico que a pesar de esta serie de influencias Rosso sea un artista original, sin embargo lo es, y en alto grado; se ha dicho de él que es "el más individualista de los artistas de su generación". " De 1513 en adelante, las pinturas de Rosso son francamente identificables.

Las mejores de sus obras fueron realizadas entre los años de 1520 y 1530, entre éstas, las más interesantes y conocidas, son las siguientes:

El descendimiento, de la Pinacoteca de Volterra. (1521).

Moisés y las hijas de Jetró. Museo de los Ufizzi. (1523).

El desposorio de la Virgen. Iglesia de San Lorenzo de Florencia. (1523).

Cristo muerto. Museo de Bellas Artes de Boston. (Aprox. 1525) .

Como puede verse, los temas de las cuatro pinturas se refieren a asuntos b́blicos o religiosos; esta circunstancia no es resultado de una libre elección del artista, sino de la demanda de este tipo de asuntos; en el tiempo de referencia la iglesia y las órdenes religiosas seguian siendo patrocinadores importantes del mercado artístico, si bien es cierto que al lado del patrocinio religioso existía también el de los mecenas cultos, que como Lorenzo de Medicis, el duque de Urbino o los Sforza de Milán, auspiciaban también otro tipo de temas, generalmente inspirados en la antigüedad clásica. Rosso dejó varios dibujos con motivos mitológicos para Pietro Aretino y otros más para ilustrar dos obras clásicas: Los amores de los dioses y Deidades antiguas.

Para ilustrar, en la medida de lo posible, el manierismo de Rosso se han tomado como ejemplos $\mathrm{El}$ descendimiento de Volterra y el Cristo muerto de Boston. Esta selección fue hecha en base a que siendo ambas obras muy representativas del manierismo en su primera fase, hay un franco corte estilístico entre ellas. Por otra parte, el conocimiento que de ellas tengo, se basa en la visión de los originales. La primera de las pinturas mencionadas tuvo su antecedente inmediato en el retablo pintado para la iglesia de Villamagna, población cercana a Volterra.

3 Linda Murray. The Late Renaissance and Mannerism. Praeguer. New York, 1967 , p. 116. 
DOI: http://dx.doi.org/10.22201/iie.18703062e.1975.44.1015

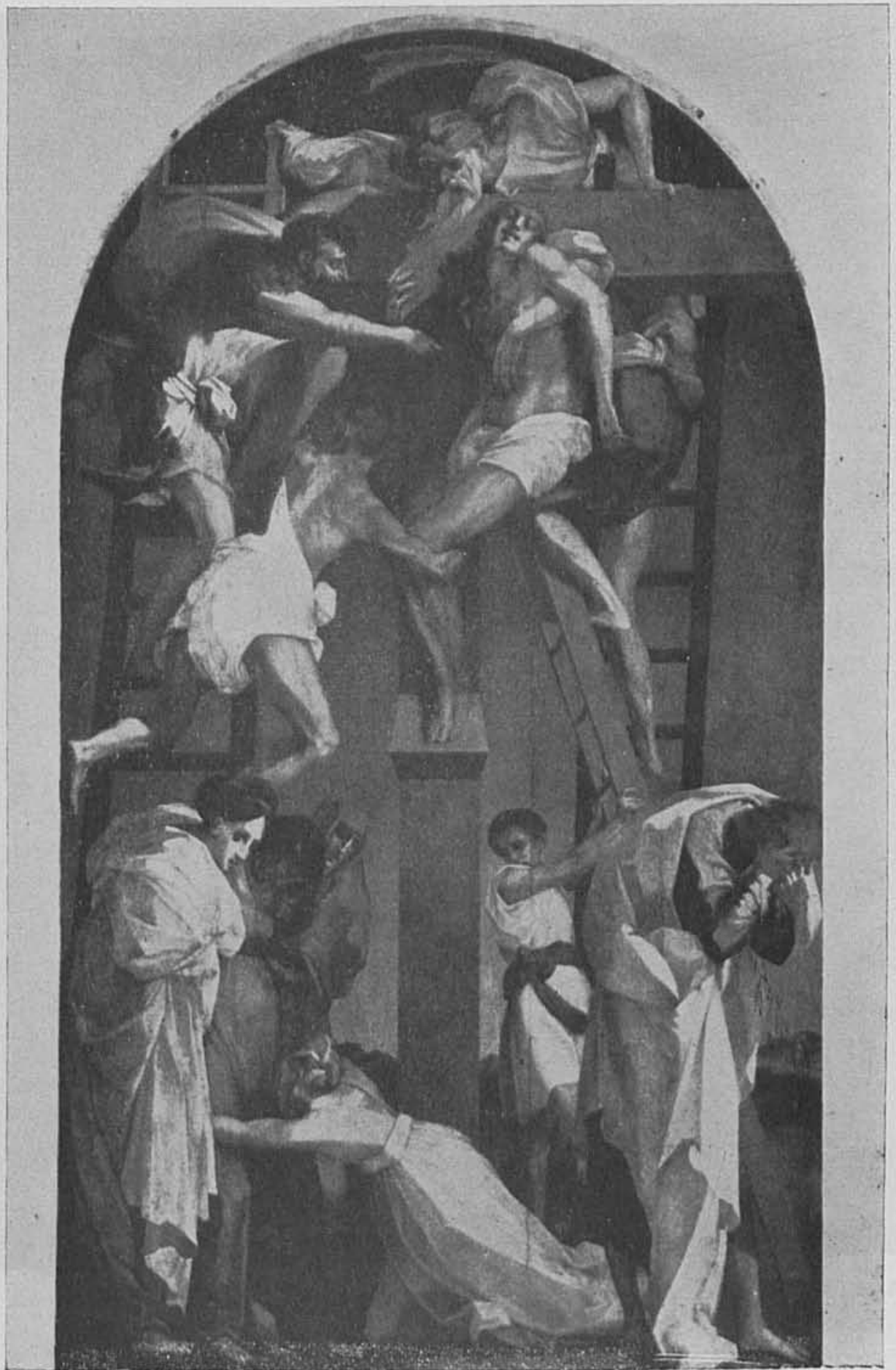

1. Rosso Fiorentino. Descendimiento, 1521, Pinacoteca de Volterra, Italia. 
DOI: http://dx.doi.org/10.22201/iie.18703062e.1975.44.1015

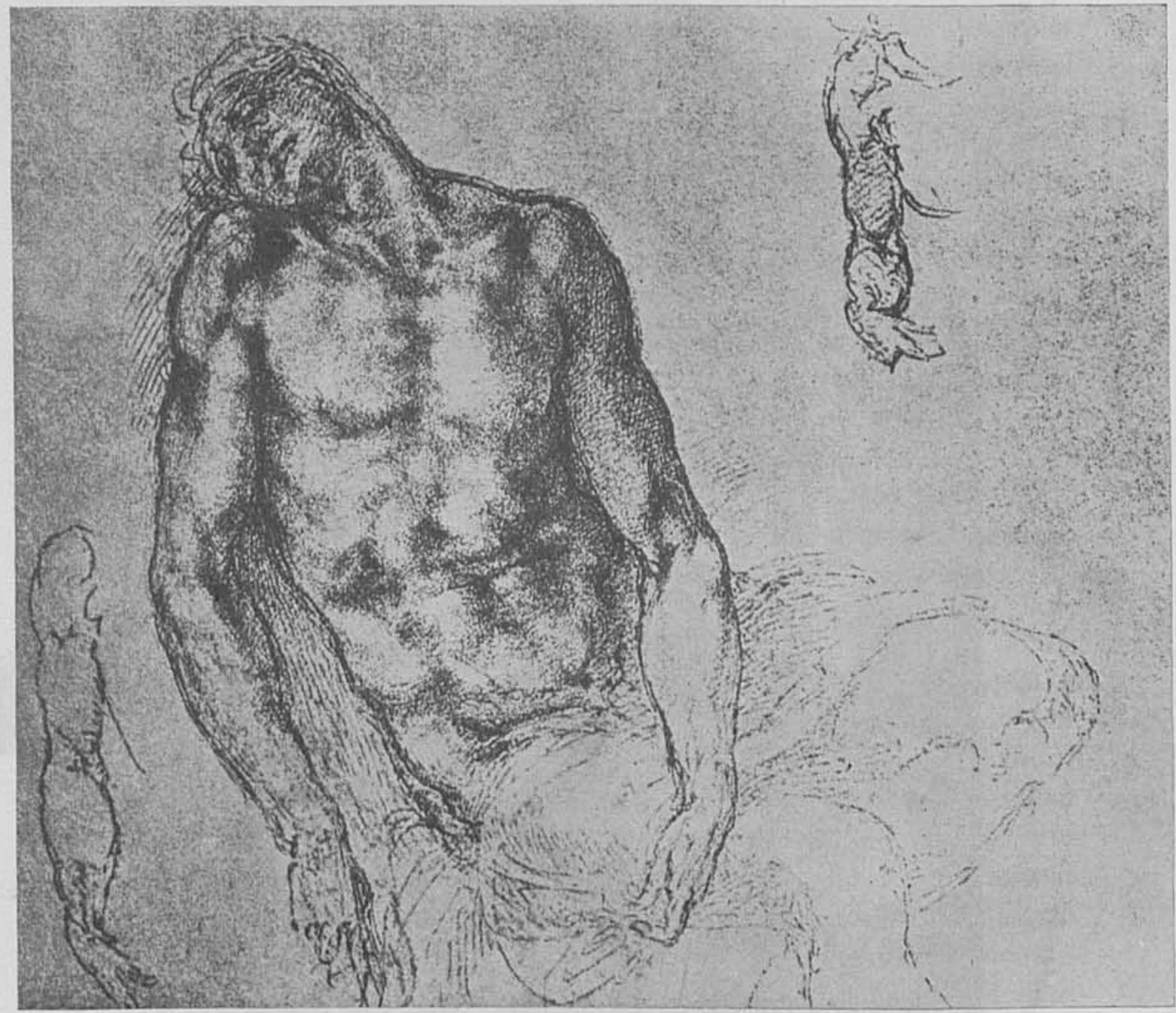

2. Miguel Ángel. Dibujo, 1519. Museo del Louvre, Paris. 
DOI: http://dx.doi.org/10.22201/iie.18703062e.1975.44.1015

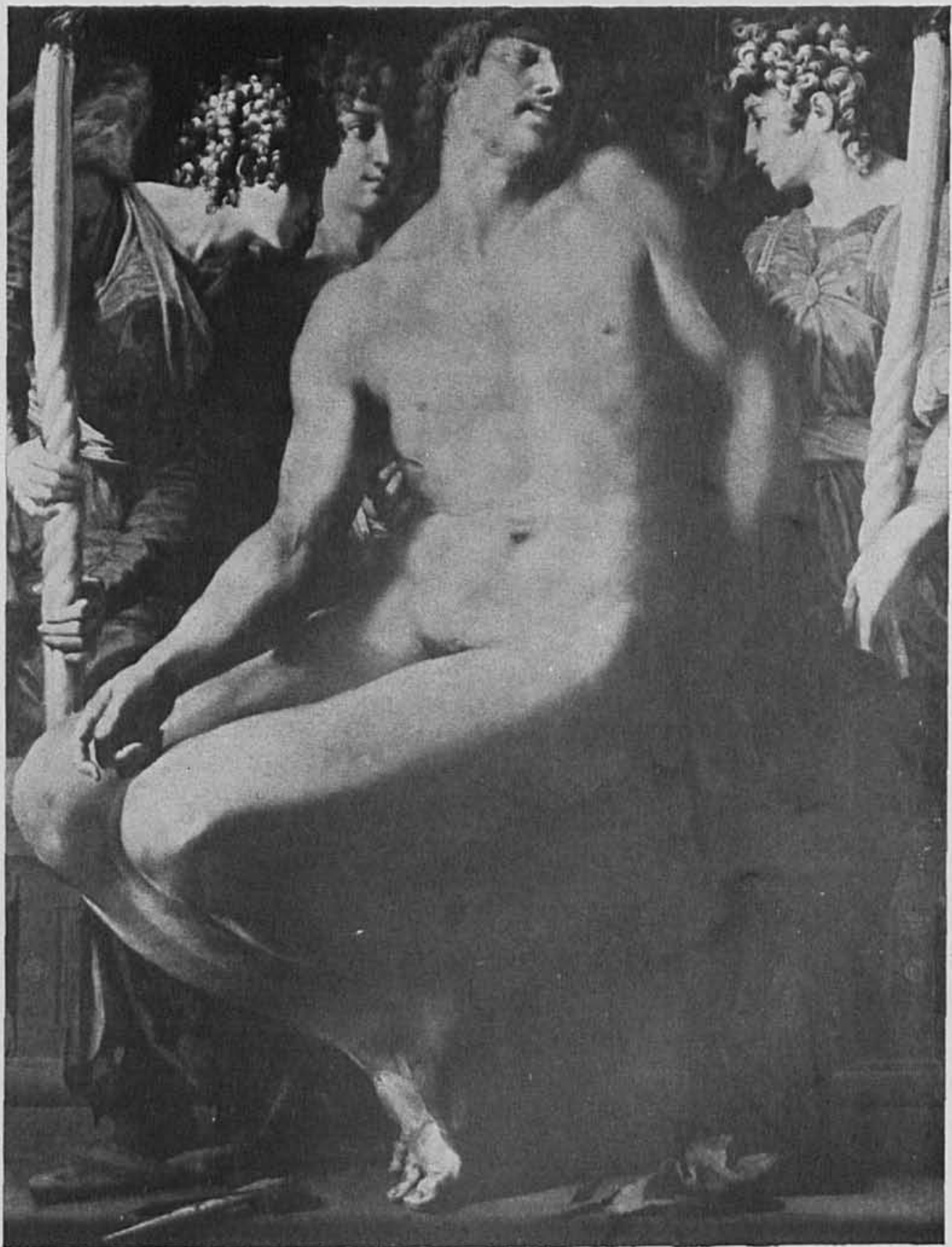

3. Rosso Fiorentino. Cristo muerto, 1524-27, Museo de Bellas Artes, Boston, U. S. A. 
DOI: http://dx.doi.org/10.22201/iie.18703062e.1975.44.1015

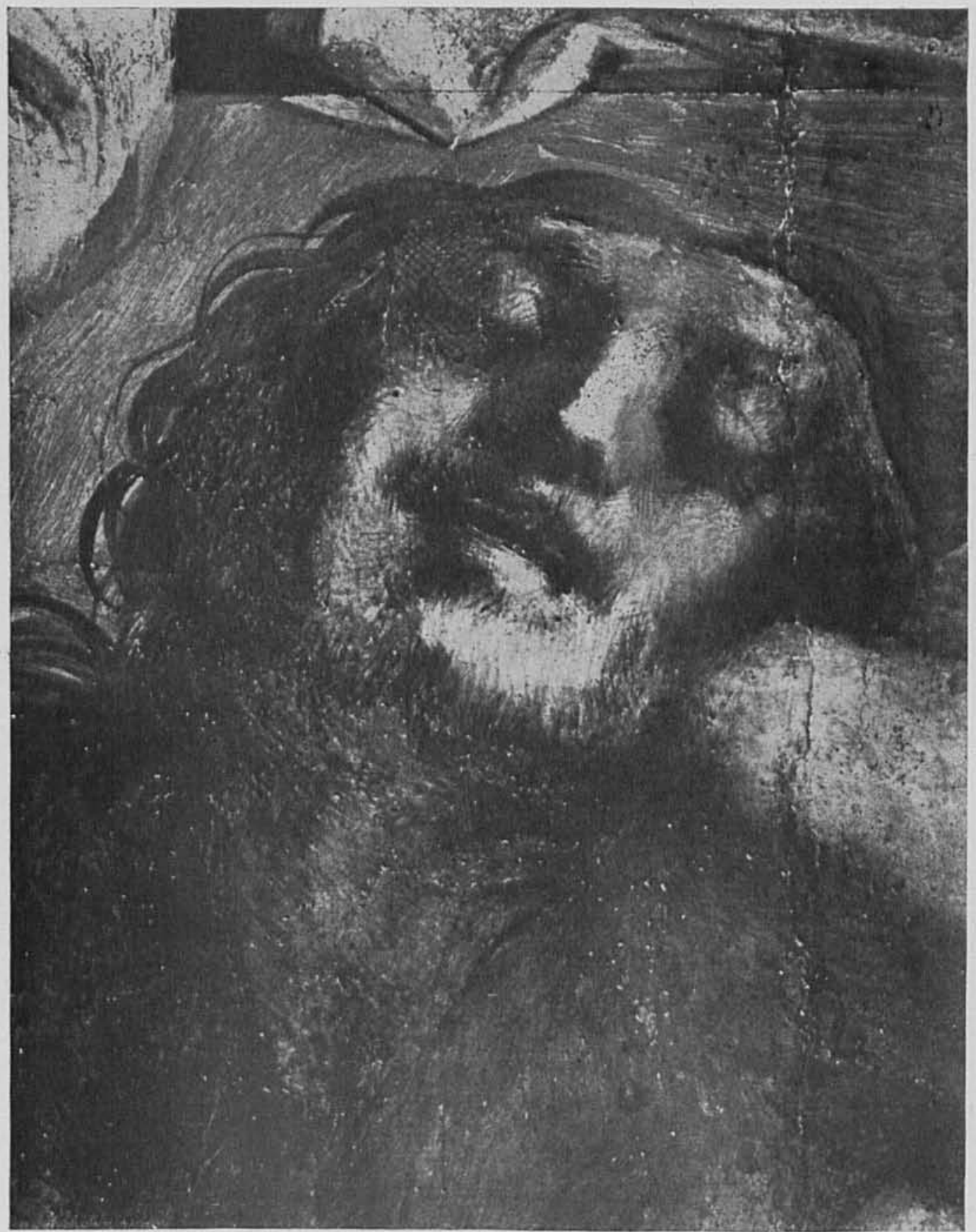

4. Rosso Fiorentino. Descendimiento, detalle. 


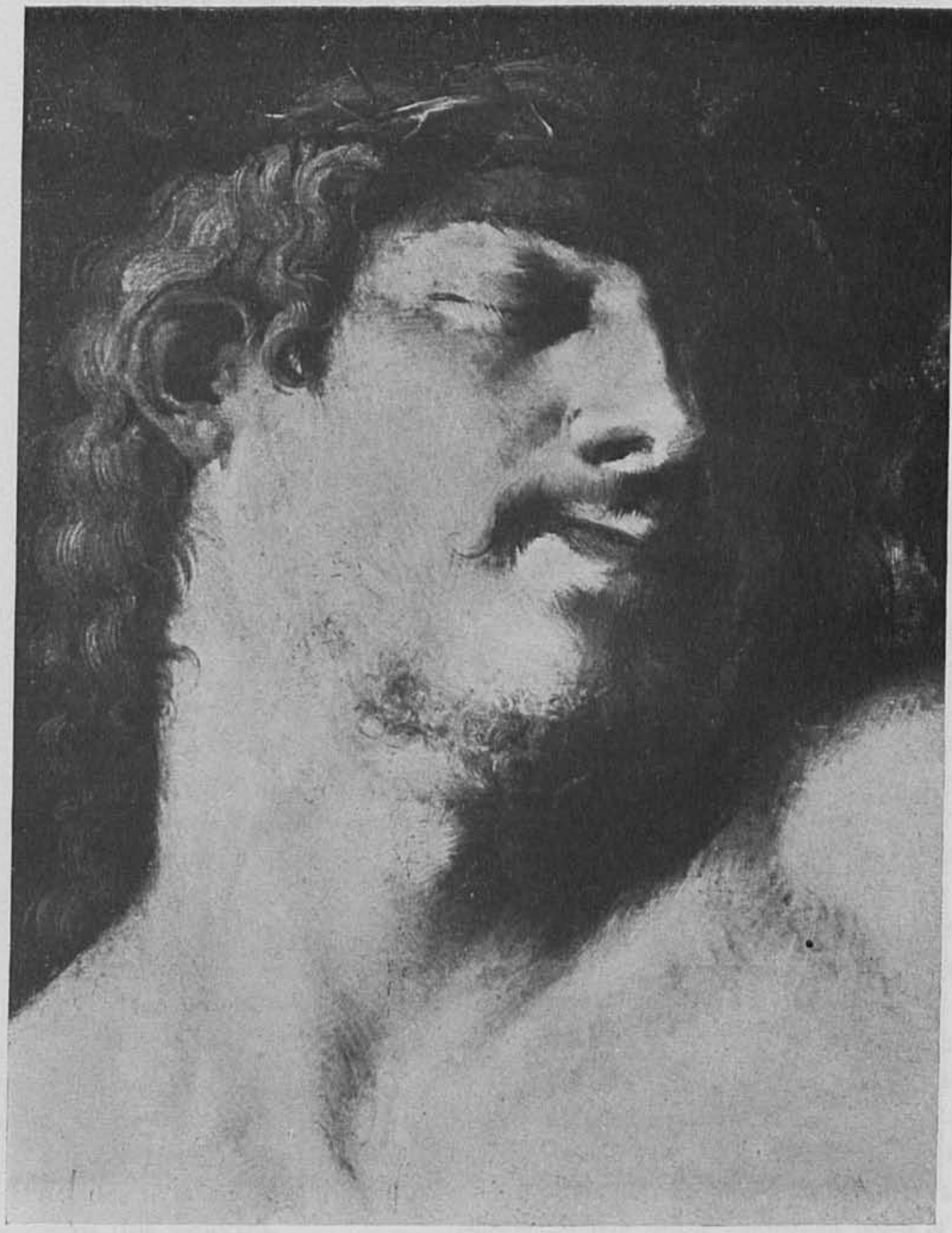

5. Rosso Fiorentino. Cristo muerto, detalle. 
DOI: http://dx.doi.org/10.22201/iie.18703062e.1975.44.1015

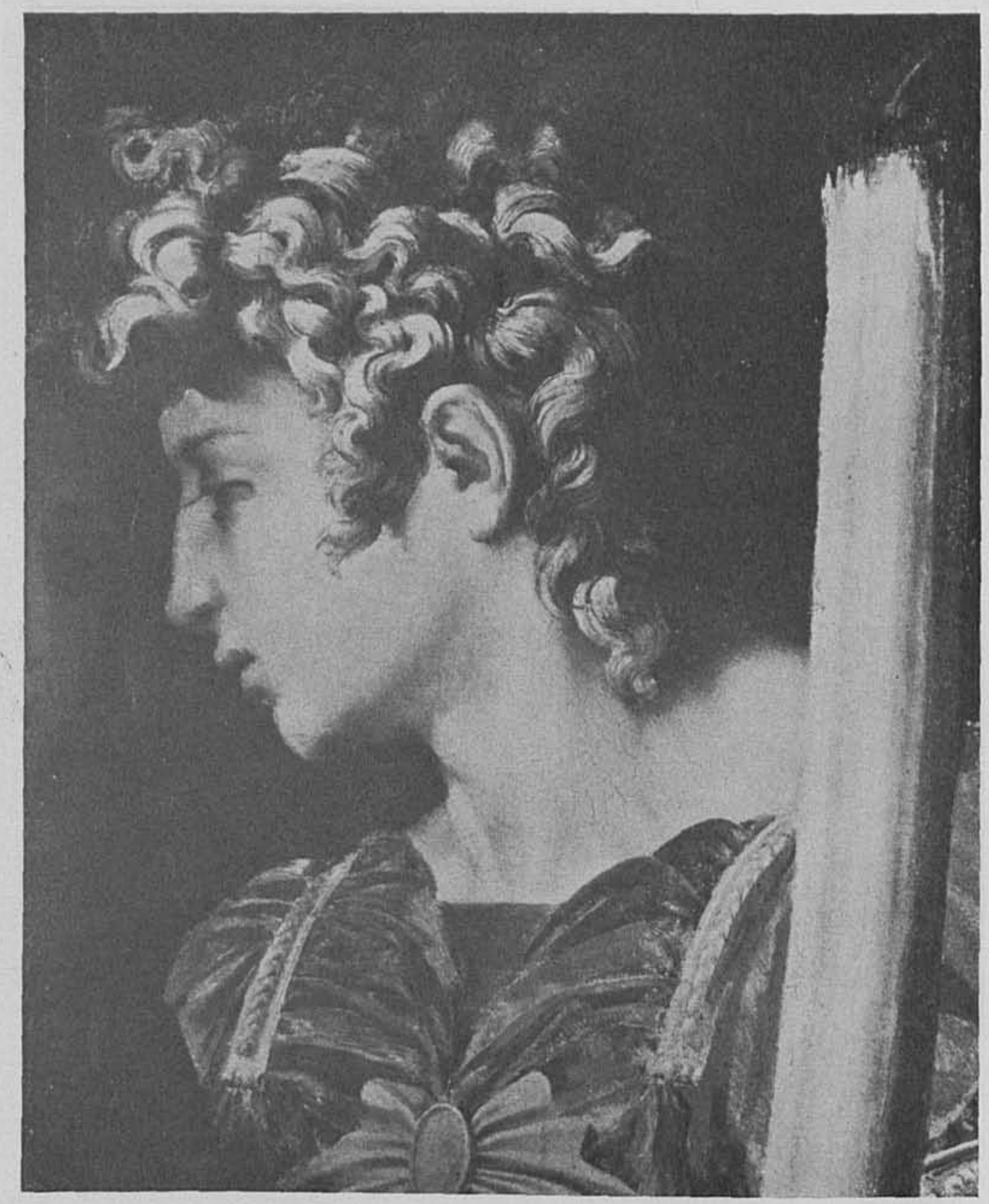

6. Rosso Fiorentino. Cristo muerto, detalle. 
Esta pintura sólo preludia levemente "la originalidad sorprendente, tan peculiar e incitante" que según Arnold Hauser 4 tiene la obra de Volterra. El fondo es monócromo, de un color azul verdoso, no el color natural del cielo, sino una tinta neutra que hace destacar vívidamente las otras tonalidades. La cruz forma un eje central que sitúa dos grupos casi paralelos al espectador, mismos que quedan divididos a la altura de los pies de Cristo. Dos escaleras situadas a ambos lados ayudan a disponer las figuras, las cuales no guardan una relación directa con la acción que se desarrolla. Los personajes no son claramente identificables y tampoco corresponden todos al lenguaje iconográfico que tradicionalmente se emplea para ilustrar este acontecimiento. En cuanto a forma de expresión, lo más característico en esta pintura es el dibujo, cortado en ángulos, geometrizado, seco, que trae a la memoria ciertos trazos de Cezánne. No existe la intención de conformar volúmenes, sino más bien se trata de un complicado juego lineal donde el tema está totalmente subordinado a la idea estética del pintor. Shermann hace derivar esta tendencia "antiplástica", de algunos relieves tardíos de Donatello, ${ }^{5}$ Hauser habla de "la inserción de formas cúbicas de apariencia inorgánica y de la transformación de los cuerpos en superficies planas", " todo ello sugiere una fuerte tendencia a lo abstracto y a la captación subjetiva de la realidad acorde con el encuentro de fórmulas plásticas que se apartan de los esquemas clásicos en aras de la búsqueda de un nuevo tipo de perfección. Linda Murray afirma que "la caracterísica prodominante de lo representado, es la impresión de dolor y pena", 7 como si la intención del artista hubiese sido suscitar en el espectador algo semejante a lo que experimentaron los protagonistas de la crucifixión real. Pero aún admitiendo que la obra es eminentemente teatral, tanto por lo que se refiere a composición, cuanto por las actitudes que guardan los personajes, más parece que Rosso quisiera provocar asombro y perplejidad, que no pena, y estas sensaciones son producidas mediante la conjunción de un colorido ultraterreno, casi espectral, con un visión lineal que favorece la percepción de las tonalidades separando planos drásticamente; la acción queda subordinada a estos dos aspectos.

4 Arnold Hauser. Pintura y manierismo. Guadarrama, Madrid, 1965, p. 110.

5 John Shearmann. "The dead Christ". Boston Museum Bulletin. Vol, LXIV, 1966, p. 159.

6 Hauser. Pintura y manierismo..., p. 111.

7 Murray. Op. cit., p. 53. 
Sicológicamente, la figura más interesante es la del propio Cristo, que guarda la actíutd de un desmayado o de un ebrio, más que la de un cadáver. La cabeza, en escorzo, está echada hacia atrás, limitando su contorno por una espesa mata de pelo rojizo; sonríe con los labios ligeramente entreabiertos, "peligrosa belleza la de la muerte", como dice el poeta inglés Gerald Manley Hopkins; peligrosa en este caso particular, porque se trata de un hombre que acaba de ser sometido a intensa tortura física, y el gesto que le ha quedado una vez que todo terminó, es más de placer que de dolor. El cuerpo, alargado y de proporciones generosas, se tuerce hacia la derecha dejando libre completamente la parte central de la cruz. Esta posición corresponde al esquema "en serpentina", tan común a muchas figuras de Miguel Angel, modalidad que torna a presentarse con insistencia en otras obras de Rosso. Existe un dibujo de Miguel Angel (hacia 1519-20), que parece haber obsesionado al artista que nos ocupa. 8 Examinándolo, efectivamente se encuentra una similitud en cuanto a posición con el del Cristo de Volterra, sin embargo las tensiones musculares y el modelado de la caja del cuerpo en el dibujo de Miguel Angel, contrastan con la morbidez y el desfallecimiento casi orgiástico del Cristo de Rosso. Las demás figuras tienen su razón de ser sólo desde el aspecto plástico, como si el horror al vacío hubiera llenado el espacio de elementos, que como la tercera escalera dispuesta en diagonal, estorban, más que ayudan, al desenvolvimiento de la acción.

Hace aproximadamente 16 años, el Museo de Bellas Artes de Boston, adquirió el Cristo muerto de Rosso, al cual se refiere Vasari en su biografía sobre este artista. "In questo tempo fece al vescovo Tornabuoni, amico suo, un quadro d'un Cristo morto sostenuto da due angeli, ch'oggi e appresso agli eredi di Monsignor della Casa, il quale fu una

8 Dicho dibujo pertenece a las colecciones del Louvre. Probablemente está inspirado en una obra escultórica helenística: el grupo de Atamante y Learco descubierto por Poelenburg durante su estancia en Roma entre 1617 y 1623 . No existe traza de la copia romana que vio Poelenburg, pero éste dejó un dibujo que reproduce exactamente la figura de Learco, donde se puede apreciar una torsión de cabeza y busto en todo análoga al dibujo del Louvre. En relación con esta misma escultura, existe otro dibujo de Miguel Angel que se conserva en casa Buonarrotti, cuya posición es la misma, con la salvedad de que se trata sólo de un torso. El dibujo aquí reproducido ha sido atribuido por Maria Vittoria Brugnoli Pace a Sebastiano del Piombo. Entre otras razones que aduce, basa su atribución en la semejanza que presenta con el Cristo de la Piedad de Ubeda, realizado por este pintor para don Ferrante de Gonzaga. María Vittoria Brugnoli Pace. "Un modello antico e due disegni attribuiti a Michelangelo." Essays presented to Rudolf Wirtkower. Phaidon Press. London, 1967, pp. 106-109. 
bellisima impresa." 9 . En el tiempo que fue realizada esta pintura, Tornabuoni, que había sido camarlengo de León $\mathrm{X}$, fue designado obispo de Borgo Sansepolcro por el Papa Clemente VII. La pintura data, aproximadamente, de 1524-1528 y fue pintada en Roma por encargo del obispo, quien tenía como emblema de su diócesis la Resurrección de Cristo. Sin embargo, el momento que capta Rosso no es propiamente la Resurrección, sino la incipiente vuelta a la vida de un cuerpo, y parece no tener antecedentes artísticos similares en la iconografía sobre este tema. Por lo general, las representaciones de Cristo sostenido por ángeles, sugieren el momento inmediato al descendimiento de la Cruz. Incluso algunas veces se presenta el calvario como fondo. El Cristo de Rosso no es el cadáver de un crucificado, ni tampoco el cuerpo glorioso del Jesús de la Resurrección. Se encuentra entre la muerte y la vida. Esto en parte puede explicarse debido a que en varias manifestaciones, tanto literarias como plásticas de este periodo, la muerte no se presenta como un evento violento y doloroso. El pensamiento de Ia muerte no aterroriza al espíritu manierista porque es visto como parte del proceso natural de la vida, como un acontecimiento más que no implica un cambio radical. O sea que está ausente "el tajante corte entre tiempo y eternidad". ${ }^{10}$ Por eso algunos héroes manieristas típicos se encuentran vivos, aún y cuando han pasado ya por el proceso de la muerte (como el padre de Hamlet), en tanto que otros participan en su vida terrenal de cierto tipo de vivencias que, supuestamente, sólo ocurren en el mundo de los muertos.

En la pintura motivo de análisis, existe un sarcófago en forma de altar y además están presentes las candelas, símbolo de resurrección. Se encuentran, en primer término, algunos instrumentos de la pasión, los clavos, la esponja para el vinagre y, asimismo, Cristo ostenta la corona de espinas, sin embargo no hay traza de sangre, ni de maltrato en el cuerpo, que presenta la pequeña herida del costado sólo como una delicada fisura en la piel.

Shearmann ${ }^{11}$ hace ver que en esta pintura ha ocurrido un cambio estilístico importante en relación al Descendimiento de Volterra, "un inesperado pulimiento y elegancia la caracteriza... el retorno a la

9 Giorgio Vasari. Vite de'pitu eccellenti pittori, scultori e architetti. Pazzini Carli. Siena, 1792. T. VI, p. 293.

10 Franzepp Wurtenberger. Il Manierismo. Silvana Editoriale d'Arte. Milano, 1964, p. 219 .

11 John Shearmann. The dead Christ, p. 161. 
plasticidad, al claroscuro modelado, lleva una nueva calidad linear, donde están ausentes los bruscos contrastes de luz y sombra y el dibujo "staccato", interrumpido, de la fase "antecedente". Resulta claro que el estilo antiplástico anterior a la llegada de Rosso a Roma se vio modificado por el trato con Miguel Angel y por el conocimiento de la escultura antigua, y es por eso que vuelve a la tactilidad, a la apriencia escultórica y al goce por las texturas y líneas de pliegue que hacen de ciertos detalles de este cuadro motivos de minucioso análisis.

Los manieristas han tenido reputación de ser muy buenos dibujantes, pero malos coloristas; este concepto es correcto sólo para la segunda generación manierista italiana, 12 ya que tanto Rosso, como Parmigianino y Pontormo manifestaron, en forma arquetípica, una renovación colorística notable en relación con artistas anteriores. Rosso emplea en este cuadro una gama de colores exquisitamente ácidos, corales, verdes crudos, turquesa, azules porcelanizados, diversos tonos de amarillo, colores que parecen extraidos de flores o de gemas y que tienen un valor intrinseco, dando al conjunto del cuadro una extraordinaria sofisticación. El cuerpo de Cristo es color marfil antiguo, ligeramente translúcido, en torno a él se desarrolla una gama riquísima de colores a través de las vestiduras de los ángeles, que no son dos, como Vasari describió, sino cuatro, si bien sólo los que están en primer término portando las velas manifiestan abiertamente su corporeidad. La figura de Cristo está presente nuevamente en serpentina, o sea que el movimiento se estructura por el alargamiento anti-natural de la caja del cuerpo y de las piernas, lo cual permite una línea continua y fluida que asume tres direcciones diferentes. Los hombros quedan en diagonal y la cabeza se vuelve hacia la izquierda. Los rostros de los cinco personajes que forman la escena, casi quedan al mismo nivel, lo cual permite apreciar el contraste entre los bellísimos efebos de faz andrógina que rodean a Cristo y el rostro del mismo, que aparece como hombre de mayor edad que sus acompañantes, con una expresión satisfecha y abandonada, mientras que ellos manifiestan franca admiración y una cierta curiosidad. No son, en ninguna forma, figuras dolientes, ni están animados de sentimientos compasivos; uno de ellos toca cuidadosamente la pequeña herida del costado, otro queda en la penumbra y los dos que se encuentran en primer término ostentan sus armónicos perfiles,

12 Jacques Busquet. La pintura manieriste. Biblioteque des arts. Neuchatel, 1964, p. 148. 
reminiscentes de las "teste divine" de Miguel Angel, en una actitud estudiadamente lánguida y elegante. $\mathrm{El}$ de la derecha se inclina hacia cristo, acercándole el rostro al cuerpo en forma tal, que si la acción tuviese secuencia, presumiblemente concluiría en un beso. Los dos grandes cirios encendidos, que por cierto no prestan ningún tipo de iluminación al conjunto, están torcidos hacia la mitad y no guardan un esquema erecto, como si la intención fuese abolir del conjunto todo aquello que no se presentara como ambiguo. Ambigua es en cuanto a expresión y en cuanto a movimiento la figura de Cristo, como asimismo lo es el porte de sus acompañantes. Para unos ojos ajenos al lenguaje tradicional ilustrativo de esta temática, el cuadro podría representar a un hombre en estado dionisíaco, más no por efectos de alcohol, sino como resultado de algo mucho más intenso. Los efebos que lo rodean admiran su belleza y a la vez se asombran de su estado; las candelas torcidas acentúan la morbidez del asunto, como también el sarcófago que bien podría simbolizar un lecho nupcial. Se ha dicho de esta pintura que es un "Laocoonte cristiano", designación que no corresponde, desde un punto de vista personal, al significado de las relaciones existentes entre los personajes que conforman el grupo de Rosso, el cual está marcado por una artificiosa sensualidad que traduce un dejo de necrofilia y homoerótica, ajeno al dramatismo convulso que anima el conjunto de padre e hijos en la obra helenística.

Esta extraña pintura queda como paradigma del estilo en su primera fase. Es revolucionaria en casi todos sus aspectos. Además, su visión produce esa sensación de obra acabada y conclusiva que reúne caracterf́sticas técnicas perfectamente asimiladas a una originalísima concepción del tema, tanto en el aspecto plástico cuanto en el simbólico.

El juicio que hace Vasari sobre Rosso corresponde en forma bastante aceptable al conocimiento que podemos tener de este artista a través de sus obras. "Il Rosso era, oltre la pintura, dotato di bellissima presenza, il modo di parlar suo era molto grazioso e grave... aveva ottimi termini di filosofia, e quel che importa piú che tutte le altre suebonissime qualitá, fú ch'egli del continuo nelle composizioni delle figure sue era molto poetico, e nel suo disegno fiero e fondato, con leggiadra maniera e terribiltá di cose stravaganti, ed un bellissimo compositore di figure." 13

18 Vasari, Op. cit., p. 288. 
Siguiendo a Vasari, además de las cualidades descritas, Rosso era grave, considerado y muy juicioso. Sin embargo, un incidente vulgar y de poca monta lo llevó a darse muerte por propia mano. "Macchiato il propio onore, decissi d'uccidersi da se stesso piuttosto ch'esser castigato da altri." 14 El asunto no pudo haber sido más banal. Encontrándose en Fontainebleau y siendo profundamente apreciado por la corte, gozando además de buenas prebendas, Rosso sufrió un robo en sus propiedades. Dicho despojo debió haber sido discreto, pues Vasari habla de unos ducados y ciertos objetos valiosos; incidentes como éste es raro que no se presenten una o más veces en el curso de una vida, en cualquier época. Rosso acusó del robo a su sirviente y discípulo, a quien parecía querer y estimar mucho. No sólo se conformó con hacer aprehender a Francesco Pellegrino, tal era el nombre del presunto ladrón, sino que dispuso que lo atormentaran "hasta que hablara", cosa que el infortunado individuo hizo con verdad, no pudiendo admitir ser culpable de algo que no había realizado. Ya hecho el mal y aclarado el asunto, que naturalmente trajo consigo enojo y malestar, pero que en virtud de la personalidad del ofensor se hubiese echado pronto al olvido, éste decidió ingerir poderosísimo veneno. Vasari cuenta que Rosso ingirió el líquido y murió pocas horas después, el 14 de noviembre de 1540, a la edad de 45 años. No tuvo un epílogo extraño si se le ve dentro de contexto; varios artistas de su generación fueron esquizoides, melancólicos o histéricos y terminaron también suicidándose. Estos casos reafirman a la época como periodo crítico en que las tensiones a las que está sujeto el humano sobrepasan la capacidad de autocontrol y al instinto de conservación. Por eso Hauser habla del manierismo como fase cultural enajenada donde impera la paradoja.

Como el fenómeno estético sigue sus propias leyes, el espíritu manierista produjo en todos los géneros obras que se cuentan entre las más preciadas de la cultura occidental. Además de ello, este periodo tuvo tal impacto sobre los estilos posteriores, que su aspecto dialéctico ha sobrevivido a través de la posteridad y sigue reapareciendo, no sólo como tendencia, la que puede ser considerada al igual que el barroco como una constante, sino como principio generador de elementos formales directamente relacionados con los conceptos estilísticos imperantes en la primera fase del movimiento.

14 Ibidem, p. 304. 
Por cuanto a Rosso concierne, su personalidad "de espiritual anarquia frente a lo que en la Florencia de su tiempo constituía convención, costumbre o regla fundada" 15 encuentra a lo largo del tiempo, correspondientes en muchos momentos, pero sobre todo en nuestro siglo. Este hombre, de existencia ambulante y aventurera, que pasó los años de su vida en Roma, en compañia de un simio al cual amaba entrañablemente, y que se suicidó en pleno periodo creativo, siendo pintor oficial de Fontainebleau, resulta un ejemplo de organización surrealista. No habla Vasari de ninguna mujer que lo hubiera amado, pese a haber sido dotado por la naturaleza de una bellísima presencia. Un crítico contemporáneo, al referirse al proceso de identificación de sus obras dice que "ofrecen un curioso muestrario de obras inconexas, propias de un espíritu indeciso y característico de un periodo tan inquieto..." 16 Aunque este juicio es inexacto, porque Rosso no es propiamente inconexo, sino fantástico, es indudable que tanto su vida como sus realizaciones, presentan el elemento irracional, imaginativo, producto de motivaciones inconscientes, que sólo se encuentran en personalidades profundamente escindidas.

15 Evelina Borea. Rosso Fiorentino. Pinacoteca de los Genios. Buenos Aires, 1964. No 102, s.p.

18 Julio E. Payró. "El juicio moderno sobre Rosso", en Rosso Fiorentino. Pina. coteca de los Genios. 